\title{
Ueber Lufteintritt in die Blutbahnen durch den puerperalen Uterus.
}

\author{
Von \\ Prof. Dr. Theodor Kézmárszky, \\ Budapest.
}

Unvorhergesehene plötzliche Todesfälle während der Geburt und im Wochenbette gehören glücklicherweise zu den Seltenheiten. Als die hauptsächlichsten Ursachen derselben werden bekanntlich Lufteintritt in die Venen des Uterus und Embolie der Lungenarterien betrachtet. Während nun die neuere Literatur eine ganze Reihe von Fällen aufweist, in denen Embolie der Lungenarterien durch Section nachgewiesen wurde, ist die Casuistik des Lufteintrittes in die Gefässe des puerperalen Uterus eine ungemein spärliche; ja, der Mangel an einschlägigen Beobachtungen aus neuerer Zeit hat dahin geführt, dass, und wie mir scheint mit Unrecht, so manche der früher veröffentlichten Fälle bemängelt oder anders gedeutet wurden. Es scheint mir daher von Interesse, einen hierhergehörigen Fall, der auf meiner Klinik beobachtet wurde, $\mathrm{zu}$ veröffentlichen, um so mehr, als derselbe geeignet ist, ältere ähnliche Fälle zu bekräftigen. An die Erzählung des Falles mögen eine kurze Uebersicht der bekannt gewordenen Beobachtungen und einige allgemeine Bemerkungen sich anschliessen.

Die 36 Jahre alte unverheirathete Wäscherin Julianne J. meldete sich den 19. März 1877, Vormittags $10 \mathrm{Jhr}$, mit mässig starken Wehen in der Gebärklinik der k. Universität, und ward unter Protokoll-Nr. 408 aufgenommen. Sie giebt an, zum vierten Male schwanger zu sein, zum ersten Male vor zehn, zuletzt vor dritthalb Jahren geboren, und ihre Periode Mitte Juli des vorigen Jahres zum letzten Male gehabt $\mathrm{zu}$ haben. Die Kreissende ist gut genährt, $151 \mathrm{Cm}$. hoch und wiegt 59,5 Kilo. Ihre schlaffen Brïste enthalten wenig 
Serum. Der grösste Umfang des Unterleibes beträgt $117 \mathrm{Cm}$. Durclı die gespannten Uteruswandungen ist über der Symphyse der Kopf der Frucht zu fühlen; andere Kindestheile lassen sich nicht deutlich erkennen. Herztöne sind nicht zu hören. Durch den zwei Querfinger weiten Muttermund touchirt man den sehr leicht beweglichen Schädel. Nach dem Befunde wurde Hydramnios diagnosticirt.

Von Mittag an wurden die bis dahin seltenen und schwachen Wehen häufiger und stärker. Etwas vor drei Uhr Nachmittags drängte sich aus der Schamspalte der in linker Seitenlage befindlichen Gebärenden die pralle, stark faustgrosse Blase hervor, weshalb die dienstthuende Hebamme den Versuch machte, dieselbe zu sprengen. Da ihr dies indessen nicht gelang, entfernte sie sich auf einige Schritte von dem Bette, um der Institutshebamme Meldung zu thun. Inzwischen war die Blase gesprungen, und die sofort an das Bett eilende Hebamme fand dasselbe ïberschwemmt mit Fruchtwasser. Etwa fünf Minuten darauf - die Hebamme war noch mit dem Aufschöpfen des Wassers beschäftigt - schnellt die Kreissende plötzlich den Kopf nach rüekwärts, schnappteinige Mal angestrengt nach $L u f t$, ihr Gesicht färbt sich livid und ihr Athem stockt. Die ganze Scene hatte, sich so blitzschnell abgespielt, dass der in kaum zwei Minuten an das Kreissbett anlangende klinische Assistent, Dr. Liebmann, keinen Puls mehr fand, zwei unvollständige respiratorische Bewegungen des Thorax wahrnahm, und nur noch die Herztöne der mittlerweile auf den Rücken gelegten Sterbenden zu unterscheiden vermochte. Während nun durch den anwesenden Praktikanten die künstliche Respiration eingeleitet wurde, legte Dr. Liebmann an den in der Beckenhöhle befindlichen Kopf unverzüglich die Zange an, und extrahirte eilends einen tief asphyktischen Knaben von 48,5 Cm. Länge und 2,780 Gm. Gewicht, dessen Belebung nicht gelang. Unmittelbar nach Abnabelung des Kindes folgte der Kuchen auf äusseren Druck. Trotz aller Bemühungen war inzwischen die Herzthätigkeit der Entbundenen erloschen, die Frau war todt. - Das aufgeschöpfte Fruchtwasser füllte zwei Schüsseln und betrug vier Liter.

Als ich etwa eine halbe Stunde nach dem Ereignisse die Verschiedene sah, fand ich ihr Gesicht mässig cyanotiseh, den Gebärmuttergrund in Nabelhöhe, den Uterus mässig hart, seine hintere Wand von etwas teigiger Consistenz. - Es warf sich selbstverständlich in erster Linie die Frage um die Ursache des plötzlichen Todesfalles auf. Dr. Liebmann gewann beim ersten Anblicke der Kreissenden den Eindruck, als ob eine Eklamptische vor ihm wäre, die gerade am Ende des Anfalles sich befindet, zu welcher Zeit bekanntlich das Aufhören der letzten Zuckungen nicht selten von der noch mehrere Secunden währenden Apnoë überdauert wird. Dem widersprach indessen die entschiedene Versicherung des anwesenden Praktikanten sowohl als der geübten Hebamme, der zufolge Convulsionen nicht vorhanden gewesen waren. Es konnte deshalb und weil sonstige Anhaltspunkte fehlten, nur an Lufteintritt oder Embolie der Langenarterien gedacht werden. 
Die 20 Stunden post mortem durch Prof. Scheuthauer ausgeführte Section ergab im Wesentlichen Folgendes: Die weichen Hirnhäute blutarm; Hirn von teigiger Consistenz, serös durchfeuchtet, mässig mit Blut versehen; in den Ventrikeln etwa $7 \mathrm{Gm}$. klares gelbliches Serum. Die sehr stark geblähten Lungen bedecken das Pericardium vollständig, sind blutarm, und enthalten mässig viel schäumendes Serum. Der linke Ventrikel des mässig zusammengezogenen Herzens enthält wenig flüssiges, gelblich blasses Blut. Im rechten Ventrikel, besonders am Ostium der Lungenschlagader, ferner in der Vena cava inferior und in den Venen des Uterus flüssiges, mit kleineren und grösseren Luftblasen gemengtes, blass rothbräunliches Blut. Auf der Schnittfläche des Uterus strömt auf Druck schaumiges Blut aus den Venen. In der Vena cava inferior sind durch Luftschichten geschiedene Blutsäulchen zu sehen, die sich hin und her schieben lassen, ohne zu confluiren. - Nach der Ansicht Prof. Scheuthauer's konnten die Luftblasen in den Gefässen nicht Product der Fäulniss sein; denn einerseits fehlten an der Leiche anderweitige Fänlnisserscheinungen, andererseits war in anderen grossen Venen, so in der $\mathrm{Ju}_{\mathrm{u}}$ gularis, keine Spur von Gasblasen zu entdecken.

Den Chirurgen war die Thatsache schon lange bekannt, dass Eindringen von Luft in die während einer Operation absichtlich oder unabsichtlich eröffneten Venen, namentlich des Halses und der oberen Hälfte des Thorax, Symptome schweren Collapses, ja plötzlichen Tod zu veranlassen im Stande sei. Dieselbe Gefährlichkeit des Eindringens von Luft in die Blutbahnen lehrte das Experiment. Um die Mitte des gegenwärtigen Jahrhunderts tauchten Mittheilungen in der Literatur auf, die es unzweifelhaft machten, dass Luft auch durch die eröffneten Venen des puerperalen Uterus in den Kreislauf gelangen und Anlass zu plötzlichen Todesfällen Kreissender oder Wöchnerinnen geben könne. Als Olshausen 1864 einen einschlägigen Fall veröffentlichte ${ }^{1}$ ), konnte er schon elf auf Gebärende und Puerperen bezügliche ähnliche Fälle zusammenstellen, bei denen die Section gemacht worden war; ferner sieben Fälle, die, obne Obductionsbefund veröffentlicht, wahrscheinlich hierher gehören. In der mir zu Gebote stehenden neueren Literatur finde ich einige weitere Beobachtungen, mit Sectionsbefunden, und ausserdem mehrere Fälle, die mit grösserer oder geringerer Wahrscheinlichkeit auf Lufteintritt durch die Venen der Gebärmutter zurückzuführen sind.

1) "Ueber Lufteintritt in die Uterusvenen." Monatsschrift für Geburts kunde 1864, 34. Bd., S. 350. 
Lufteintritt in die Blutbahnen durch die Venen des Uterus ist an zwei Bedingungen geknüpft: es müssen vorerst offene Venen in der Gebärmutter vorhanden sein, dann muss Luft in den Uterus gelangen. Während der Geburt, sowie in der ersten Zeit des Wochenbettes ist die erste der Bedingungen immer vorhanden: die Placentarstelle bietet offene Venensinus dar, die in den ersten Tagen des Puerperiums nur durch lose haftende Thromben geschlossen sind. In der Schwangerschaft kann Lösung der Placenta, in späterer Zeit des Wochenbettes Lockerung und Herausfallen eines Thrombus dieselben Verhältnisse herbeiführen. Zur Beleuchtung der zweiten Bedingung - Eindringen von Luft in den Uterus - und des Lufteintrittes in die Venen lohnt es sich wohl der Mühe, die bisher bekannt gewordenen Fälle gruppenweise nach ihren thatsächlichen ätiologischen Momenten durchzusehen.

Vorerst wären die Fälle zusammenzufassen, in denen Luft gewaltsam in den Uterus gepumpt wurde. Hierher gehören folgende:

1. Depaul ${ }^{1}$ ) wollte bei einer Viertgeschwängerten im siebenten Monate durch Uterindouche die Frühgeburt einleiten. Dabei wurde die Canüle $1 \mathrm{Cm}$. vom Muttermunde gehalten. Nachdem sich wiederholt ein gurgelndes Geräusch in der Vagina eingestellt hatte, nahm Depaul die Canüle heraus and liess die Schwangere aufstehen. Doch nach zwölf Minuten war sie eine Leiche. Bei dem eilends ausgeführten Kaiserschnitte quoll aus der Uteruswunde blutiger Schaum; ebenso fand sich zwischen Ei und Uteruswand Luft in zahlreichen Blasen.

2. Olshausen liess bei einer Zweitgebärenden (Zwillingsschwangerschaft) nach mehr als 24 stündiger Daver der Geburt behufs Beschleunigung der Eröffnung der fingerweiten Cervix mittels Clysopompes die warme Uterusdouche anwenden. Als der Apparat zum dritten Male etwa acht Minuten lang gewirkt hatte, fing die Kreissende an über Beklemmung zu klagen. Das Mutterrohr wurde aus der Vagina entfernt. Die Kreissende richtete sich im Bette in die Höhe, fiel aber sogleich besinnungslos aus demselben heraus und verschied in höchstens einer Minute unter einigen zuckenden Respirationsbewegungen und Verzerrung des Gesichtes. Fünf Minuten nach dem Tode wurde beim Betasten des Leibes ein weit verbreitetes Knistern bemerkt. - Die Section (acht Stunden post mortem) ergab: In den Coronargefässen des Herzens, im rechten Herzen, in der Cava inferior viel Luftblasen; der Uterus knistert überall beim Streichen seiner Wandungen mit der Hand, eine Menge seiner Gefässe

1) s. Olshausen's Artikel: „Nachtrag“. 
mit Luft gefüllt. Retroperitoneales Emphysem nach rechts. Die eine Placenta in kleinerer, die andere in bedeutenderer Ausdehnung abgelöst.

3. Litzmann ${ }^{1}$ ) erzählt folgenden Fall. Nachdem bei einer zum sechsten Male Schwangeren behufs Einleitung der Frühgeburt wegen engen Beckens durch einen elastischen Katheter warmes Wasser zwischen Ei und Uteruswand obne Erfolg injicirt worden war, wurde mittels Mayer'schen Pumpapparates die Douche applicirt. Bei der vierten Douche lag die Schwangere, wie gewöhnlich, auf dem Geburtsstuble in halb sitzender, halb liegender Stellung, und das Injectionsrohr wurde vom Assistenzarzte bis an den hoch hinaufgezerrten Muttermund eingeführt, und ausdrücklich darauf geachtet, dass die Spitze desselben nicht in den Cervicalkanal selbst eindrang. Nach reichlich zehn Minuten wurde das Rohr plötzlich durch mehrere heftige Hustenstösse abwärts gedrängt, das Aussehen der Sehwangeren zejgte sich etwas verändert, und sie antwortete auf Befragen, ,sie glaube, sie befinde sich übel". Inzwischen war die Douche sofort abgebrochen. Aber schon hatte die Schwangere das Bewusstsein verloren, das Gesicht färbte sich immer livider, die Hände wurden kiihl, der Puls kaum fühlbar, das Athmen wurde immer seltener und erlosch nach einigen stossweisen Inspirationen mit einem tiefen krampfhaften Athemzuge ganz. Sectionsbefund (16 Stunden post mortem): Beim Betasten des Leibes ist deutlich Crepitation wahrnehmbar. Beim Einschneiden der äusseren Bedeckungen des Thorax ergiesst sich aus den Venen ein grossblasiges, stark lufthaltiges Blut. Mit Ausnahme der Venen des Darmkanales und der Pfortader fast alle Unterleibsvenen zum Theil prall mit Luft gefüllt (Cava dünndarmdick); ebenso einige Venen am Grunde und an der vorderen Fläche der Gebärmutter. Im rechten Herzen grossblasiges schaumiges Blut, - Placenta adhärent, bis auf einen kleinen, circa $5 \mathrm{Cm}$. im Duxchmesser haltenden Abschnitt ihres unteren Randes, unterhalb dessen ein etwa handtellergrosses lufthaltiges Coagulum zwischen Uterus und Eihäuten.

Es unterliegt wohl keinem Zweifel, dass in jedem der drei angeführten Fälle Luft mit der Injectionsflüssigkeit in den Uterus gelangte. In Depaul's Falle war während der Injection ein gurgelndes Geräusch vernommen worden; Litzmann bemerkt ausdrücklich, dass laut nachträglicher Untersuchung neben dem nicht genau schliessenden Stempel Luft in die Pumpe eindrang; Olshausen hält es für allbekannt, dass bei einer Clysopompe, zumal wenn sie nicht träglich gebraucht wird, sehr leicht ausser dem Wasser auch Luft durch das Saugrohr aspirirt und dann mit ausgespritzt wird. In keinem der Fälle findet sich ein sicherer Anhalt dafuir, dass das Ende des Rohres in die

1) Dieses Archiv, II. Bd., S. 178. 
Cervix gedrungen wäre. Ja Litzmann sowohl als Depaul heben das Gegentheil hervor, Olshausen schliesst die Möglichkeit des Eindringens nicht aus, da bei der Application die Hebamme eingestandenermassen das Mutterrohr während der ganzen Dauer der Injection nicht selbst gehalten hatte. Wenn danach in 01shausen's Falle die Möglichkeit nicht ausgeschlossen werden kann, dass die Injection des lufthaltigen Wassers direct in den Uterus geschah, so scheinen die beiden anderen Fälle zu beweisen, dass der auch nur in die Vagina gepumpte Wasserstrahl im Stande ist, durch die Cervix in das Cavum uteri zu dringen. In allen drei Fällen war die Kraft des Strahles eine so grosse, dass partielle Lösung des Kuchens bewirkt wurde. Es liegt kein Grund vor, die vorgefundene partielle Trennung der Placenta in Olshausen's und Depaul's Falle einer anderen Ursache zuzuschreiben. In Litzmann's Falle war allerdings Injection zwischen Eihäute und Uterus vorangegangen; allein weder das durch den Katheter rückfliessende Wasser hatte blutige Beimischung, noch zeigte sich später Blutung, so dass wohl mit ziemlicher Wahrscheinlichkeit angenommen werden kann, die Placentartrennung sei hier auch durch den Wasserstrahl herbeigeführt worden. Wemn nun in zwei Fällen der einströmende Wasserstrahl ohne Zweifel, im dritten wahrscheinlich kräftig genug war, die Placenta partiell zu trennen, so liegt die Annahme doch wohl am nächsten, dass die mit Luft bläschen gemischte Flüssigkeit auch direct in die eröffneten Venensinus zu dringen vermochte.

Eine ähnliche Beobachtung hat W in ckel der gynäkologischen Section der Hamburger Naturforscherversammlung mitgetheilt. ${ }^{1}$ ) Es wurde in der Nachgeburtsperiode wegen starker Blutung mittels Ballonspritze Liquor ferri gegen die Portio vaginalis gespritzt. Gleich darauf starb die vorher ohnmächtig gewordene Frau. Es fand sich Luft in der Cava inferior; ebenso waren die Venẹn der Herzwand mit Luft gefüllt.

Mit grosser Wahrseheinlichkeit ist der von Olshausen citirte Fall John Swinburne's auch hierher zu rechnen.

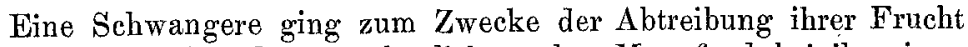
zu einer Frau, bei der sie plötzlich starb. Man fand bei ihr einen Gummikatheter, der wahrscheinlich zum Lufteinblasen beniitzt wor-

1) Dieses Archiv, X. Bd., S. 386. 
den war. Die Section ergab Luft im rechten Herzen, in der Jugularis, in den.Venen und Sinus des Uterus; die unverletzten Eilhänte sammt Placenta partiell gelösst; an der Innenfläche des Uterus eine Perforationsöffnung, direct mit einem Sinus communicirend. - In diese Perforationsöffnung war Swinburne's Ansicht nach der Katheter gedrungen und auf diese Weise Luft in die Vene geströmt oder eingeblasen worden.

Mit Wahrscheinlichkeit sind ferner einige Beobachtungen hierher zu zählen, bei denen unter ähnlichen Umständen plötzlicher Tod oder beängstigende Erscheinungen aufgetreten waren, die jedoch des Beweises der Obduction entbehren.

Spiegelberg ${ }^{1}$ ) machte in der achten Schwangerschaft einer 33 jährigen Frau wegen allgemein verengten platten Beckens die künstliche Frühgeburt. Gegen Ende der dritten Douche klagte die Schwangere plötzlich über Schwindel, Schmerz in der Herzgrube, tiefe Beklemmung. Das Gesicht wurde leichenblass und schweissbedeckt, der Ausdruck angstvoll, Athmung mühsam, beschleunigt. Grosse Unruhe; Puls fadenförmig, zuweilen verschwindend. Schïttelfrost, gefolgt von schwerem Collaps; Dyspnoë und Unruhe nehmen $\mathrm{zu}$, die Kranke wirft sich stöhnend auf dem Bette umber - ein Bild tiefsten Verfalles. Erst ganz allmälig trat Erholung ein. Spiegelberg (s. auch dessen Lehrbuch, S. 659) deutet die geschilderten Erscheinungen als Luft- resp. Luft- und Blutembolie von den Uterusvenen ausgehend. Die Luft war eingepumpt. Zwar war das Ansatzrohr der Douche zuvor luftleer gemacht, aber anstatt erst durch einen schwachen Wasserstrahl die bereits in der Scheide befindliche oder mit der Hand eingeführte Luft zu entfernen, hatte die Hebamme sofort aus bedeutender Höhe - mehr als $4 \mathrm{M}$. - den kräftigen Wasserstrahl gegen das untere Uterinsegment gerichtet und infolge dieses hohen Druckes ein Ruickweichen der Luft aus der Scheide verhindert. Wasser und Luft wurden nun durch den Cervicalkanal, der für einen Finger bequem durchgängig war, in die Uterushöhle zwischen Eibäute und Uteruswand eingepumpt und bewirkten, nach der reichlichen Blutung zu schliessen, jedenfalls eine nicht ganz unbedeutende Ablösung der Placenta. Dadurch war die Bildung frischer Thromben an dieser Stelle und des Uebertrittes von Luft in die Venen gegeben; die wechselnde Action des Uterus bewirkte dann das Weitere.

Ferner führt Olshausen eine Reihe von Beobachtern an, die nach Injectionen Beängstigung, Ohnmacht, beschleunigten Puls u. s. w. auftreten sahen. Der bekannte'Fall Scanzoni's, in welchem nach Einpumpen von Kohlensäure in die Cervix einer

1) Wiener, Zur Frage der künstlichen Frühgeburt bei engem Becken. Dieses Archiv, Bd. XIII, Hft. 1, S. 94. 
Schwangeren unter plötzlich auftretendem Starrkrampf erschwerte Respiration u. s. w., endlich nach etwa anderthalb Stunden der Tod erfolgt war, und bei dem sich Lösung des unverletzten Eies, sonst hingegen nichts Positives durch die Section ermitteln liess, lässt sich wohl auch nicht leicht anders als durch Gaseintritt in die Circulation deuten. - Wynn Williamss) erwähnt, dass ihm zwei Fälle zur Kenntniss gekommen wären, in welchen bei einer Injection von warmem Wasser Luft in die Uterinvenen eindrang und der Tod erfolgte. Nach Barnes ${ }^{2}$ ) beobachtete Simpson plötzlichen Tod einige Minuten nach Injection atmosphärischer Luft. Von Interesse ist endlich, dass Litzmann's Schwangere während ihrer vorhergegangenen fünften Gravidität unter ganz ähnlichen Umständen schwere analoge Zufälle durchzumachen hatte. Bei der dritten Application der Douche (Mayer'sche Pumpe) musste nach kaum 15 Minuten aufgehört werden wegen plötzlicher Ohnmacht der Sch́wangeren mit erschwerter Respiration, cyanotischer Gesichtsfärbung, Kälte der Haut, kaum fühlbarem Pulse, aus welchem Zustande sie mühsam wieder geweckt wurde. Litzmann führt diese alarmirenden Symptome mit vollem Rechte unbedingt auf Eintritt von Luft in die Uterusvenen zurück.

Eine zweite Gruppe bilden diejenigen Fälle, in denen der Lufteintritt in die Gebärmutter resp. in die Blutbahnen während der Geburt oder im Wochenbette spontan erfolgte. Das Verständniss dieser Fälle wird durch diejenigen Kenntnisse vermittelt, die wir über die Saugkraft des Uterus und die Veränderlichkeit des intraabdominellen Druckes besitzen. Schon Simpson macht darauf aufmerksam, dass der Uterus während seiner Erschlaffung (,Wehenpause") die in der Vagina befindlichen Flüssigkeiten ansauge. Nach Schatz'3) experimentellen Untersuchungen ist bei ruhenden Bauchdecken der intraabdominelle Druck je nach der Körperlage beträchtlichen Schwankungen unterworfen; er sinkt stufenweise, je nachdem der Körper in Rückenlage sich befindet, oder dabei der Steiss höher liegt als der Brustkorb, oder wenn der Rücken zu oberst ist (Knieellbogenlage). Tiefe Inspiration verringert gleichfalls den intraabdominellen Druck. Bei ruhenden Bauchdecken wird der intraabdominelle

1) s. Olshausen's Artikel: „Nachtrag".

2) Lectures ou obstetric operations. London 1870.

3) Dieses Archiv, IV. Bd.: „Beiträge zur physiologischen Geburtskunde". 
Druck hauptsächlich durch das Gewicht des Thorax und der Bauchdecken beeinflusst, so dass derselbe wächst, wenn Thorax und Bauchdecken über (Rückenlage), hingegen sinkt, sobald jene unter der Bauchhöhle (Bauchlage) sich befinden. Wenn im letzteren Falle der Thorax tiefer liegt als der Steiss (Knieellbogenlage, Knieschulterlage), so kann der intraabdominelle Druck geringer werden als der Atmosphärendruck (unteratmosphärischer Druck). Dies letzte Gesetz bildet bekanntlich theilweise die physikalische Basis derjenigen Operationen, die in Knieellenbogenlage ausgeführt zu werden pflegen (Nabelschnurreposition, schwere Wendung).

Hegar ${ }^{1}$ ) wendet diese Gesetze auf das bekannte Aspirationsphänomen der abdominellen Hohlorgane folgendermassen an. In Knieellbogenlage oder in Sims'scher Seitenlage sind die Bauchdecken sowohl als der Scheideneingang gleichem Atmosphärendruck ausgesetzt. Da jedoch die Bauchdecken am tiefsten liegen, so müssen sie vermöge ihres Gewichtes einen Theil des auf sie wirkenden atmosphärischen Druckes aufheben. Es wird daher der auf die Bauchdecken wirkende atmosphärische Druck um das Gewicht der Bauchdecken geringer sein als derjenige Druck, der auf das Vaginalostium lastet. Das Gleichgewicht ist infolge dessen gestört: das Gewicht der herabsinkenden Bauchdecken hat die Tendenz, die sich berührenden beiden Vaginalwände von einander zu trennen; die äussere Luft trachtet durch den Scheideneingang in die unter geringem Drucke stehende Vaginalhöhle zu gelangen, oder mit anderen Worten, die Vagina aspirirt die äussere Luft. Was hier der Einfachheit halber von der Vagina und den Bauchdecken gesagt wurde, gilt natürlich auch einerseits von der Urethra und dem Rectum, andererseits von den zu unterst liegenden Wänden der Hohlorgane selbst („Gewicht der Gewebe"). Gewisse Hülfsvorrichtungen sind im Stande, das Ansaugen zu verhindern: jungfräuliche oder enge Scheide, deren Wände mit ihren Schleimhautfalten fest aneinander liegen, der Hymen oder dessen übereinander gefaltete Lappen, endlich dor Schliessmuskel. Wenn diese Hülfsvorrichtungen fehlen oder unzulänglich sind, was in eminenter Weise während oder nach der Geburt der Fall ist, oder wenn der Verschluss durch äussere Einwirkungen gelockert wird, wenn beispielsweise durch Körperbewegung dic ein-

1) „Saugp̣hänomene am Unterleibe.“ Dieses Archiv, Bd. IV, S. 531. 
ander berührenden Schleimhautfächen verschoben werden, oder der Verschluss gar durch einen eindringenden fremden Körper (Hand, Instrumente) aufgehoben wird, so kann die auf den Scheideneingang lastende atmosphärische Luft leicht das noch fehlende ergänzen, d. h. den völligen Durchbruch bewerkstelligen, und muss nun so lange in die Vagina einströmen, bis das Gleichgewicht wieder hergestellt ist.

Aehnlich, wie unter gewöhnlichen Verhältnissen die Vagina, verhält sich der Uterus während und unmittelbar nach der Geburt, ja selbst in der späteren Zeit des Wochenbettes. Bildet doch die Gebärmutter während der Geburt mit der Vagina einen continuirlichen Schlauch, und diese Continuität bleibt auch noch im Puerperium lange bestehen, wozu noch kommt, dass in Folge der wechselnden Contraction und Relaxation des Uterus thatsächlich Pumpwirkung erzielt wird. Hegar illustrirt die Saugkraft der Gebärmutter mit einer Beobachtung von nahezu experimentellem Werthe. Bei einer in Knieellbogenlage Kreissenden zögerte die Ausstossung des Rumpfes nach Geburt des Kopfes zu lange. Es wurde der Fundus gehoben und gerieben, endlich ein leichter Zug in der Achselhöhle ausgeführt. Das Kind wurde geboren, und sogleich stürzte ein Strom von Luft mit brodelndem Geräusche in den Uterus, woher sie sodann sammt Nachgeburt und Blut in Rückenlage herausgepresst wurde.

Nach all dem Angeführten glaube ich den auf meiner Klinik beobachteten Fall auf folgende Weise erklären zu müssen.

Zur Zeit des Blasensprunges befand sich die Gebärende nahezu in Sims'scher Seitenlage, d. h. sie lag mit angezogenen Schenkeln auf der linken Seite, ihr Rumpf schräg über dem Bette, ihr Kopf nahe am Rande zweier flacher Rosshaarpolster derart, dass das Gesicht und die Brustfläche etwas nach abwärts sahen. In der beschriebenen Lage befindet sich der ausgedehnte Unterleib, namentlich seine höchste Wölbung um ein Weniges tiefer als das am Rande des Bettes ruhende Becken, jedenfalls merklich tiefer als das Vaginalostium. Es ist leicht verständlich, dass in dieser Lage unter 'sonst günstigen Verhältnissen der intraabdominelle Druck ein negativer werden konnte. Diese günstigen Verhältnisse stellten sich in dem Augenblicke ein, als mit Abfluss des Fruchtwassers Uterus und Bauchdecken beinahe plötzlich in Ruhezustand versetzt wurden, und zwar um so sicherer und vollständiger, als wegen des vorhandenen Hydramuions der Inhalt des Uterus respec- 
tive der Abdominalhöhle um ein Bedeutendes geringer werden musste. Mittlerweile hatte die vor der Schamspalte erscheinende Fruchtblase die ohnehin weite Scheide der zum vierten Male Gebärenden ausgedehnt, die Schamspalte zum Klaffen gebracht. Sowie nun die Eihäute barsten und das Wasser abfloss, folgte Scheide sowohl als auch Vaginalostium nicht rasch genug durch Zusammenziehung und Collabiren dem plötzlichen Schwinden ihres Inhaltes, sondern beide blieben noch in dem Augenblicke klaffend, als der intraabdominelle Druck bereits negativ geworden war. Das Resultat dieser Umstände musste natürlich Aspiration. der mit der Gebärmutterhöhle frei communicirenden Luft von Seite des Uterus sein.

Welchen Weg die einströmende Luft nehmen konnte, ist auch nicht schwer aus folgenden Betrachtungen zu eruiren. Sowie die aus der Scheide faustgross sich hervordrängende Blase geborsten war, collabirten auch ihre schlaffen Wände, die Eihäute. Es musste demnach zwischen Eihäuten and Scheidenwand ein Zwischenraum entstehen, den der eindringende Luftstrom auf Kosten der Eihäute vergrössern half. $\mathrm{Da}$ nun der Zusammenhang zwischen Eihäuten und Uterus, wie aus dem tiefen Herabdrängen der Fruchtblase leicht erklärlich, in nicht unbeträchtlicher Ausdehnung gewiss gelöst sein musste, so konnte selbstverständlich die durch die Gebärmutter aspirirte Luft nur zwischen Eihäute und Uteruswand eindringen. In dem ungefähr fünf Minuten währenden Zeitraume, der vom Blasensprunge bis zum ersten Symptome der Athemnoth verstrich, verkleinerte sich der Uterus durch Retraction und accommodirte sich seinem nun geringeren Inhalte. Gleichzeitig sank der bisher hochstehende und bewegliche Kopf auf den Muttermund, dessen Ränder sich an ihn anschmiegten, und verlegte auf diese Weise die Muttermundsöffnung. Vielleicht schon während dieser Uterusretraction, ohne Zweifel hingegen unter der darauf folgenden activen Contraction des Fruchthalters, musste theilweise Lösung der Placenta erfolgen, was nicht auffallen kann, wenn die nothwendig eintretende, sehr bedeutende Volumensverkleinerung der Gebärmutter nach plötzlichem Abflusse einer ungewöhnlich grossen Menge Fruchtwassers ins Auge gefasst wird. Das Fehlen von Blutung kann nicht als Gegenbeweis gelten, weil der herabgesunkene Kopf den Muttermund tamponirte und wegen Kürze der Zeit das im Uteruscavum angesammelte Blut sich nicht Bahn brechen konnte. Nach alledem 
fand die nächste We folgende Verhältnisse: zwischen Eihäute und Uteruswand ein gewisses Luftquantum; auf der Haftstelle des theilweise gelösten Kuchens offene, mehr weniger klaffende Gefässlumina; den Muttermund durch den herabgesunkenen und zum Theil herabgepressten Kopf verlegt. In dem Mäasse, als nun die beginnende Contraction das intrauterine Luftquantum in höhere Spannung versetzte, musste dasselbe nach derjenigen Richtung strömen, in der ein Ausweg vorhanden war, das heisst, die Luft musste in die offenen Venen der Placentarstelle eindringen. Diese Erklärung scheint dem Umstande am besten zu entsprechen, dass zwischen Lufteintritt in den Uterus und Erstickungsnoth ein Zeitraum von ungefähr fünf Minuten verstrich, und dass die Symptome der Athemnoth am Ende dieses Zeitraumes thatsächlich mit einer kräftigen Treibwehe coincidirten, die im Stande war, den Kopf in die Beckenhöhle zu befördern.

Olshausen führt folgende, dem mitgetheilten ganz oder grossentheils analoge Fälle an:

L evy beobachtete bei einer fiebernden 50jährigen Gebärenden gleich nach dem spontanen Blasensprunge, ohne dass Blutung vorhanden war, collabirtes bäulich bleiches Gesicht, kalte Extremitäten, kaum fühlbaren Puls, ängstliches Schnappen nach Luft. Während der Extraction des Kopfes mittels der Zange starb die Kranke. Bei der 20 Stunden post mortem gemachten Section ergab sich, ohne dass Fäulniss vorhanden gewesen wäre, in den Unterleibsvenen schäumendes Blut, besonders viel Luft in der rechten Vena spermatica interna, in den Venen der Lungen und des ïbrigen Körpers. Placenta grösstentheils gelöst.

Wintrich berichtet ibber eine Kreissende, die nach der Geburt des Kindes und theilweiser Lösung des Kuehens plötzlich unter Convulsionen und Suffocationserscheinungen gestorben war, ohne dass eine bedeutende innere Blutung oder dergleichen erfolgt war, was als Todesursache hätte gelten können. Bei der Section fand sich im rechten Herzventrikel schaumiges Blut.

In G. May's Falle war eine Frau in der Geburt gleich nach dem Blasensprunge plötzlich gestorben. Die Section ergab eine Ausdehnung des rechten Herzohres durch Luft.

Vielleicht gehört auch der Fall von Duvernoy hierher; doch liegt einerseits der Verdacht auf Verblutung vor, andererseits kann Bildung von Fäulnissgasen nicht ausgeschlossen werden (hohe Lufttemperatur, Section 24 Stunden post mortem); überdies ist die Todesart unbekannt, da die Person todt aufgefunden wurde. - Die Beobachtung, die Olshausen als dritten Fall 
Bruler's anführt (plötzlicher Tod unte leichten Zuckungen, gleich nach dem Blasensprunge) lässt sich auch, trotzdem dass das Obductionsergebniss fehlt, am ungezwungensten auf Eindringen von Luft in den Kreislauf zurückführen:

Von ganz besonderem Interesse ist endlich der von Geo. Cordwent veröffentlichte Fall ${ }^{1}$ ), zu dessen Erklärung ausser den oben angeführten theilweise auch noch andere Factoren herangezogen werden müissen. Der Fall ist folgender:

Bei einer 28 jährigen gesunden Kreissenden waren vor Aukunft des Arztes starke Treibwehen aufgetreten, und auf ihr Verlangen wurde ihr erlaubt, in aufrechter Stellung zu bleiben. Nach wenigen heftigen Wehen wurde ein schöner Knabe geboren, der, auf den Boden stïrzend, die ganze Placenta mit sich riss. Beinahe unmittelbar darauf wurde von der Umgebung eine Art von Gurgeln gehört; es konnte indessen nicht gesagt werden, ob dasselbe von Gurren im Darme herrühren mochte. Die Patientin blieb etwa eine Minute wie früher in aufrechter Stellung und hielt sich an dem Bettpfosten fest. Dann rief sie: „Ich sehe nicht; ich werde ohnmächtig; legt mich zu Bette!" Man kam ihrem Verlangen nach, und sie verschied augenblicklich. - Bei der 24 Stunden post mortem gemachten Section fand man den Uterus äusserlich normal, mit Ausnahme einer etwa fünf-shilling grossen Stelle am Fundus, die etwas weniges geschwellter erschien als die iibrige Oberfläche; auf Incision entwichen Luftbläschen. Placentarfläche nicht verletzt, Uterushöhle enthielt blos ein kleines Coagulum. Die Coronarvene des Magens war prall durch Luft ausgedehnt und blutleer mit Ausnahme eines dünnen Fadens, welcher der Visceralwand derselben anlag. Die rechte Seite des Herzens fand sich leicht gebläht, und als man das Herzrohr punctirte, entwichen Luftbläschen dem Blutinhalte desselben.

Der vorliegende Fall scheint mir treffend das zu illustriren, was Hegar zum Schlusse seines Artikels „Saugphänomene am Unterleibe“ sagt. Hegar erwähnt zunächst der Aspirationskraft der Blase, wenn in Rückenlage bei ruhenden Bauchdecken der Urin durch den Katheter unter kräftigem Drucke auf die Blasengegend ausgespritzt und dann plötzlich der Druck aufgehoben wird; - eine Erfahrung, die auch ich aus verschiedenen, in den ersten Tagen des Wochenbettes gemachten Beobachtungen bestätigen kann. Zur Erklärung der Erscheinung fügt Hegar hinzu: „Die Schwere (sc. der Bauchdecken und des Thorax) kommt hier nicht in Betracht. Vermöge der Elasticität der comprimirten

1) St. George's Hospital Reports, Vol. VI, 1871-1872. London J. et A. Churchill. 
Gewebe kommt es zu einer rückgängigen Bewegung. Es bildet sich lebende Kraft, wodurch die Gleichgewichtslage überschritten und die Einsaugung veranlasst wird. Ob der Druck und dessen Sistirung mittels der Hand geschieht oder ob eine starke Action contractiler Theile, wie der Bauchdecken, rasch aufhört, bleibt sich gleich."

Allerdings gesteht nun Hegar in seiner „Berichtigung und Nachtrags“ ') zu obigem Artikel, er sei nicht im Stande gewesen, durch directe manometrische Versuche den experimentellen Nachweis seines angeführten Satzes zu liefern; allein dies gilt nur für reine Rückenlagen. Ueberdies hebt er ausdrïcklich hervor, es sei eben nur der positive Beweis nicht zu erbringen, und es seien das Experiment störende Nebeneinflüsse durchaus nicht auszusehliessen gewesen. - Ich möchte deshalb den angeführten Satz H egar's schon deshalb ohne jedes Bedenken auf Cordwent's Fall anwenden, weil die Verhältnisse in demselben von den bei Hegar's Versuchspersonen vorhandenen doch wesentlich verschieden waren. Vorerst stand die Kreissende aufrecht (,the patient remained... standing as before, and holding at the bed-post"). Aus der Beschreibung ist allerdings die Stellung nicht genau zu entnehmen, es liegt indessen die Vermuthung nahe, es sei die Haltung eine etwas nach vornüber gebeugte gewesen; darauf deutet wenigstens der Umstand hin, dass die Kreissende sich doch wohl mit den Händen an den Bettpfosten stützte. Vielleicht war also selbst die Stellung der Kreissenden eine für die Aspiration günstige, d. h. mit etwas überhängenden Bauchdecken. Nach einigen heftigen Wehen wurde das Kind ausgestossen und riss auf den Boden fallend die ganze Nachgeburt mit sich (,dragged with it the whole placenta"). Es ist wohl nicht anzunehmen, dass kräftige Treibwehen einer gesunden und starken Gebärenden nicht energische Action der Bauchpresse im Gefolge gehabt hätten, und ebenso naheliegend ist, dass mit dem Aufhören des Widerstandes, d. h. dem Hervorstürzen des Kindes, auch das Mitpressen plötzlich sistirte, was wieder eine rückgängige Bewegung des elastischen Uterusgewebes sehr einleuchtend erscheinen lässt. Dieses „, $\mathrm{Zu}$ rückfedern" des Uterus konnte durch einen anderen Umstand nur erhöht werden: das auf den Boden auffallende Kind riss die ganze Plancenta mit sich. Wieder liegt die Annahme sehr nahe,

1) Dieses Archiv, V. Bd., S. 177. 
dass partielle Adhäsionen des Kuchens erst während des Sturzes, durch Spannung resp. Zerrung des Nabelstranges, getrennt wurden, was aus leicht begreiflichen Gründen ein Herabzerren der elastischen Placentarstelle bis zu einem gewissen Grade, und eben deswegen ein stärkeres ,Zurückfedern" derselben bewirken musste. Wenn nun noch hinzugerechnet wird, dass höchst wahrscheinlich dem heftigen Drängen wohl unmittelbar eine tiefe Thoraxinspiration folgte, so scheinen alle diese Umstände das Zustandekommen eines unteratmosphärischen Druckes in der Bauch- resp. Uterushöhle und Aspiration von Luft hinlänglich zu erklären. Die Aspiration scheint in Cordwent's Falle sogar eine so mächtige gewesen zu sein, dass die Luft in Folge derselben nicht blos in das Cavum uteri, sondern direct in die Venen gelangen konnte. Darauf deutet wenigstens hin, dass zwischen Lufteindringen und den ersten Symptomen der Athemnoth blos ein Zeitraum von ungefähr einer Minute (about a minute) verstrich, ein Zeitraum, während dessen die eingedrungene Luft ihren Weg durch das rechte Herz in die Lungengefässe zurücklegen konnte. Aehnliche hochgradige Spannung und directes Eindringen des Luftstromes in die Venen müssen in den oben angeführten Fällen angenommen werden, in denen die bedrohlichen Symptome dem möglichen Eindringen der Luft in den Genitalkanal unmittelbar (,gleich“) folgten; und es würde sich der eindringende Luftstrom dieser Annahme nach ähnlich verhalten, als ob derselbe eingepumpt worden wäre.

Den angeführten reihen sich diejenigen Beobachtungen an, in welchen Lufteintritt einige Zeit nach der Geburt oder im Verlaufe des Wochenbettes erfolgt war. Von den durch Olshausen citirten Fällen gehören hierher:

Lionet's Fall, in welchem während und nach der natürlichen Geburt sich grosse Schwäche zeigte. Drei Stunden nach der Niederkunft grosse Blässe, erschwertes Athemholen, Würgen, unregelmässiger Puls; keine innere Blutung. Tod fünf Stunden post partum. Section (30 Stunden post mortem): In den Herzventrikeln nebst geringer Menge Blut einige Luftblasen, mehr auf der rechten als auf der linken Seite. Luft in einigen Hirnvenen. Verfasser glaubt Fäulniss ausschliessen zu können.

G. May erwähnt zweier Fälle, in denen sechs Stunden resp. acht Tage post partum Tod eintrat. Man fand im ersten Falle Luft im rechten Herzohr; im zweiten Luft in der Vena cava inferior, Vena portarum und schaumiges Blut im rechten Herzen. 
Simpson beobachtete einen durch Section bestätigten Fall bei einer Wöchnerin.

In Hervieux's Falle ward am elften Tage des normalen Wochenbettes eine Einspritzung von Kamillenthee in den Uterus gemacht, worauf Frostanfall und Metrorrhagie. Am selbigen Abend während zorniger Aufregung plötzlicher Tod. Section 30 Stunden post mortem (Juli): Luft in der Vena cava inferior, in beiden Herzventrikeln, besonders dem rechten. Iliacae und Venen des Uterus enthielten keine Luft.

Ausser diesen von Olshausen angeführten Beobachtungen bemerkt Playfair'), Mc. Clintock erwähnte sechs, La Chapelle hingegen zwei Fialle, die wahrseheinlich auf Lufteintritt in die Uterusvenen zurückzuführen seien. Als sehr wahrscheinlich auch hierhergehörig berichtet derselbe Autor über einen Fall Graily Hewitt's. ${ }^{2}$ ) Es trat der Tod kurz nach der Entfernung. einer adhärenten Placenta ein, während welcher Operation Playfair's Ansicht gemäss ohne Zweifel Luft in das Uteruscavum leicht eindringen konnte. Die Symptome, "heftiger Schmerz in der Präcordialgegend, unregelmässige Respiration (distress as regards respiration) und Pulslosigkeit," seien mit denen bei Obstruction der Pulmonalgefässe identisch. G. Hewitt führt den Tod auf Shock zurück, der sicherlich im Allgemeinen nicht ähnliche Symptome hervorrufe.

Endlich theilt $\mathrm{Hegar}{ }^{3}$ ) eine interessante Beobachtung Bis ch of $\mathrm{f}^{\prime} \mathrm{s}$ (Basel) mit. Eine durchaus gesunde Wöchnerin plaudert am Morgen des fünften Tages mit ihren Nachbarinnen, lacht laut, fängt mitten jm Lachen an das Gesicht zu verziehen, mit den Gliedern zu zucken, wird blass, röchelt einige Male und stirbt nach einigen Minuten. Bei der 28 Stunden post mortem (Juli) angestellten Section fand sich aušser starken Todtenflecken sonst keine Fäulnisserscheinung. Im Herzen und den Pulmonalarterien dünnflüssiges Blut, das im rechten Ventrikel und im rechten Pulmonalstamm stark schaumig ist. Luft in den Gefässen der weichen Hirnhäute.

Die Bedenken, die Hegar gegen die Beweiskraft dieses Falles für Lufteintritt in die Gefässe ausspricht, sind ohne Zweifel gewichtig, und es liegt namentlich sehr nahe, das Vorhandensein von Luft im Herzen und in den Gefässen auf Fäulniss zurückzuführen. Allein abgesehen davon, dass ausser starken Todtenflecken sonst keine Fäulnisserscheinungen vorhanden waren, wie erklärt es sich, dass nur im rechten Herzen und nur im rechten Hauptstamme der Pulmonalarterie Luft sich vorfand, wäh-

1) A Treatise on the Science and Practice of Midwifery, by W. S. Playfair. Vol. II, S. 352. London, Smith, Elder et Co., 1876.

2) Ebendaselbst S. 354 .

3) Dieses Archiv, V. Bd., S. 179.

-Arehiv f. Gynäkologie. Bd. XIII. Hft. 2. 
rend dieselbe links fehlte? Wenn überdies der Sectionsbefund auch keinen Anhaltspunkt über die Stelle des Lufteintrittes in das Gefässsystem bietet, so ist, wie Hegar selbst hervorhebt, der sonst vollständig negative Befund in Beziehung der Todesursache von Bedeutung. Aehnliche Bedenken, wie gegen den angeführten Fall lassen sich gegen die Beobachtung Lionet's und. Hervieux's geltend machen. In Lionet's Falle ist überdies der Umstand verdächtig, dass der Tod erst zwei Stunden nach Beginn der bedrohlichen Symptome eintrat. Nun tritt nach allem, was wir aus klinischen Erfahrungen (namentlich der Chirurgen) und Experimenten wissen; in Folge von Lufteintritt in die Circulation sehr schnell der Tod ein, oder die eingedrungene Luftmenge wird sehr bald durch das Blut absorbirt und ebenso rasch mittels Diffusion in die Lungenluft befördert, womit selbstverständlich die normalen Circulationsverhältnisse wieder hergestellt sind. In Lionet's Falle müsste entweder angenommen werden; die einmal eingedrungene Luft sei im Verlaufe zweier Stunden nicht zur Absorption resp. Diffusion gelangt, oder es hätten successive Nachschübe von Luft stattgefunden, und es wäre demnach der Tod nicht infolge des hohen Grades, sondern der langen Dauer des Pulmonalisobstruction durch Luftembolien erfolgt; - zwei Annahmen, von denen mir keine besonders grosse Wahrscheinlichkeit zu haben scheint, und ich gestehe, dass mir der Fall trotz des Sectionsbefundes mehr den Eindruck von Embolie der Pulmonalis durch einen losgelösten Thrombus macht. - Der Fall Hervieux müsste übrigens unter Voraussetzung seiner Hierhergehörigkeit ätiologisch der Beobachtung Bisch off's angereiht werden; in diesem kam Aspiration während des Lachens, in jenem während zorniger Aufwallang zu Stande. Dort wie hier kann partieller Verschluss der Glottis während tiefer Thoraxinspiration sehr gut angenommen werden, was nach Schatz ein sehr erbebliches Sinken des intraabdominellen Druckes zu Folge hat. Ueberdies macht in Hervieux's Falle auch die Stelle des Lufteintrittes keine Schwierigkeit; während der Injection in die Gebärmutter war augenscheinlich ein Thrombus losgelöst worden (Metrorrhagie), und der nachträgliche Verschluss des Gefässes konnte bis zum Abend desselben Tages gewiss nicht die zur definitiven Schliessung erforderliche Solidität erlangt haben. Nach Analogie des Beobachtung Bischoff's scheint mir diese Erklärung plausibler als die Winckel's, der zufolge die Luft in Hervieux's Falle wahrscheinlich durch die Intrauterininjection in 
die Gebärmutter gelangt war; das lange Verweilen der Luft im Uterus wäre ohne jegliches Symptom schwer verständlich. - In May's und Simpson's Fällen, derer nur ganz im Allgemeinen Erwähnung geschieht, kann selbstverständlich nicht einmal der Versuch einer Erklärung gemacht werden.

Wenn nun nach alle dem Angeführten allerdings zugegeben werden muss, dass ein bew ei sender Fall für Lufteintritt in die Gefässe des Uterus während des Puerperiums nicht vorliegt, so wird denn doch in Erwägung der Aehnlichkeit des Verlaufes sowohl als der Sectionsberichte die Wahrscheinlichkeit in der angeführten Deutung der Beobachtungen nicht wohl von der Hand gewiesen werden können, um so weniger, als ja in den Bedingungen für Lufteindringen zwischen den jetzt verhandelten und denjenigen Fällen, die als beweisende betrachtet werden müssen, nur ein gradueller Unterschied besteht.

Endlich ist hier noch der Fälle Erwähnung zu thun, in denen das Eindringen von Fäulnissgasen in die Gefässe des Uterus und in den Kreislauf im Allgemeinen constatirt wurde. Zwei Beobachtungen, die Winckel im zweiten Bande seiner „Berichte und Studien" 1) veröffentlicht, gehören hierher. In beiden Fällen hatten sich während protrahirter Geburt nach Abfluss des Fruchtwassers jauchiger Ausfluss und Fäulnissgase in der Gebärmutter entwickelt, dabei septisches Fieber mit Schüttelfrösten. Im ersten Falle erfolgte vier Stunden nach Extraction der Frucht unter Collapserscheinungen und besonders starker Cyanose der Tod. Die Section ergab nebst Perforation der hinteren Uterinwand und $400 \mathrm{Gm}$. blutig gefärbter Flüssigkeit in der Bauchhöhle, Gas in den Venen des Uterus, der Milz und des Herzens, besonders der rechten Hälfte desselben, . . S Spermatica dextra und Cava inferior durch Luft ballonartig aufgetrieben. - Es. ist nicht angegeben, nach Verlauf welcher Zeit post mortem die Obduction gemacht wurde, so dass postmortale Luftentwickelung im Blute nicht mit Sicherheit auszuschliessen ist. Erwähnt ja doch Birch-Hirschfeld (der die Section ausführte) selbst ausdrücklich ${ }^{2}$ ): „Mit dem weiteren Fortschritte der Fäulniss findet oft sehr reichliche Luftentwickelung im Blute statt (in gewissen Fällen bereits wenige

1) 0. c. "Zur Pathologie der Schwangerschaft und der Geburt," von Osterloh. S. 79 .

2) Lehrbuch der pathologischen Anatomie, S. 1285. 
Stunden nach dem Tode, z. B. bei an Septicaemie Verstorbenen).“ - Im zweiten Falle war unter hochgradigem Fieber und jauchigem Ausflusse nach wiederholter Perforation der Schädel endlich mittels Kranioklast extrahirt worden, und dem Kinde waren jauchige Flüssigkeit und zahlreiche, sehr übelriechende Gase gefolgt. Nach der Entbindung wurde in den Wänden des Uterus beim Reiben deutlich emphysematisches Knisterrasseln wahrgenommen. Unter Cyanose Tod eine Stunde post partum. Section (23/4 Stunden post mortem): Aus den Uterusgefässen quillt schaumigblutige Flüssigkeit hervor; Cava inferior, beide lliacae und mehrere Beckenvenen mässig mit Luft gefüllt; aus den Gefässen der Leber quillt ebenfalls schaumiges Blut.

Wenn nun auch in dem ersten Falle die Möglichkeit des Lufteintrittes in die Blutbahnen nicht geleugnet werden kann, im zweiten hingegen das factische Zustandekommen dieses Ereignisses zugegeben werden muss, so scheint es mir doch, als ob der Tod nicht a usschliesslich, ja nicht einmal hauptsächlich auf ,Eindringen von Luft in die Venen des Uterus und des übrigen Körpers" zurückzuführen sei, wie die Aufschrift der beiden Beobachtungen anzeigt, und wie Osterloh im ersten Falle anzunehmen scheint, im zweiten aber ausdrücklich hervorhebt. Beide Beobachtungen sind augenscheinlich Fälie von acuter Vergiftung durch Aufnahme septischer Stoffe in das Blut, und es scheint mir durch nichts bewiesen, dass diese septischen Stoffe in gasförmig flïssigem Medium suspendirt, nicht aber in tropfbar flüssigem Vehikel in die Blutbahnen gelangten. Allerdings ist auch die Möglichkeit des ersteren nicht zu leugnen, ja nach dem zweiten Falle ist dies sogar mehr als wahrscheinlich; allein es liegt kein Symptom vor, welches beweisen würde, dass die eingedrungenen Gase ausschliesslich als solche tödtlich gewirkt hätten, es müssten denn „Cyanose“, „ziemlich beträchtliche Dyspnoë“, „steigende Respirationszahl" oder Collapserscheinungen nach der Entbindung als solche Symptome angesehen werden, - Erscheinungen, die nahe zum tödtlichen Ende einer acuten septischen Vergiftung nichts Ungewöhnliches bieten. Nun kann aber schliesslich gewiss nicht geleugnet werden, dass das im ersten Falle mögliche, im zweiten hingegen bewiesene Eindringen von Gasen in die Blutbahnen durch Behinderung der Circulation mit zur Herbeiführung des lethalen Ausganges beigetragen und denselben beschleunigt haben konnte. Es wäre demnach dem Angeführten 
gemäss in beiden Beobachtungen nicht die ,A Aufnahme von Fäulniss gasen “ im Allgemeinen, sondern die ,Aufnahme von fauligen Gasen". und fauligen Stoffen überhaupt in erster Reihe hervorzuheben. - Jedenfalls ergiebt sich aus diesen Erörterungen, dass die beiden Beobachtungen Winckel's nicht den ,unerwarteten" Todesfällen im puerperalen Zustande zugezählt werden können. Es scheint mir, als ob der Fall Ramsbotham's, den Olshausen am Schlusse seines Artikels mittheilt, ähnlich beurtheilt werden köninte, wie die soeben besprochenen zwei Beobachtungen Winckel's.

Nach dem Angeführten liesse sich das, was über Lufteindringen in die Blutbahnen durch die Gefässe des puerperalen Uterus thatsächlich vorliegt, in Folgendem kurz resumiren.

Luft kann rein oder mit Flüssigkeit gemengt mittels Einpumpens oder ähnlicher Operationen in den Uterus und in dessen eröffnete Gefässe gelangen. Von grossem praktischen Interesse ist, dass erstens ein mässiger Druck, wie ihn die gebräuchlichen Injections- oder Doucheapparate auszuïben vermögen, hinreicht; und zweitens, dass die Injection durchaus nicht in die Gebärmutter selbst gemacht werden muss, sondern dass unter günstigen Umständen der blos in die Vagina geleitete Strahl im Stande ist, durch den Cervicalkanal zwischen Ei und Uterinwand und in offene Gefässe letzterer zu dringen. Spontaner Lufteintritt kann erfolgen, sobald bei offener Passage des Genitalkanales der intrauterine resp. intraabdominelle Druck geringer wird, als der atmosphärische, in welchem Falle Uterus sowohl als Uteringefässe Aspiration ausiuben. Rasche, theilweise (Blasensprung) oder gänzliche (präcipitirte Geburt) Entleerung des Uterininhaltes während der Geburt schafft die erwähnten Verhältnisse; günstige Position der Gebärenden erhöht die Disposition (Seitenlage, aufrechte Stellung). Die durch den Uterus aspirirte Luft dringt entweder direct in die offenen Gefässe, oder eine nachfolgende Contraction presst bei mittlerweile verlegtem Muttermunde die in dem Carum uteri befindliche Luft in die Venen. Höchst wahrscheinlich wirken ähnliche Verhältnisse auch im Puerperium aspirirend; tiefe Thoraxinspiration (Lachen, zornige Aufwallung) scheint $\mathrm{zu}$ disponiren: Fäulnissgase im Uterus können sich in der Geburt ähnlich verhalten, wie eben aspirirte Luft.

Aus Allem, was wir über Lufteintritt in die Venen des puerperalen Uterus wissen, ergiebt sich, dass von einer eigentlichen 
Therapie dieses Ereignisses keine Rede sein kann. Was wir zu thun im Stande sind, beschränkt sich auf Wiederbelebungsversuche. Um so dringender sind prophylaktische Massregeln zu beobachten, und diese wieder in verschiedener Richtung, je nach dem Mechanismus, des Lufteindringens.

Einpumpen von Luft wird am sichersten vermieden, wenn Apparate gebraucht werden, die einen continuirlichen Wasserstrahl durch Wassersäulendruck liefern. Ein solcher Apparat ist der einfache Wundirrigateur. Ich benutze seit etwa drei Jahren in meiner Klinik Apparate, deren Reservoir aus einem zwei Liter fassenden Glascylinder besteht. Unmittelbar über dem Boden des Gefässes befindet sich ein seitliches Spundloch, in welches vermittels eines Stöpsels aus vulcanisirtem Kautschuk ein kurzes Glasröhrchen eingepasst ist, welches wieder dem einen Meter langen Kautschukschlauche genau eingeschoben ist. Das Scheidenrohr ist entweder aus Hartgummi, mit Absperrhahn aus demselben Materiale, oder, wie neuerlich allgemein, aus Glas. Der von mir benutzte Apparat hat vor solchen mit Metallireservoir den Vorzug, leichter rein gehalten werden zu kömnen und von differenten Flüssigkeiten (Eisenchlorid) nicht angegriffen zu werden, während die Dicke des Glases ihn nicht leicht zerbrechlich macht und sein Preis mässig ist. - Wird das Scheidenrohr nicht früher eingeführt, als bis das Wasser in reinem Strahle rinnt, und entfernt man dasselbe aus den Genitalien, bevor das Niveau des Wassers im Reservoir die Ausflussöffnung erreicht hat, so kann man Miteinspritzen von Luft sicher vermeiden.

Es wurde erwähnt, dass bei geringem intrauterinen Drucke die eingeführte Hand der Luft den Weg bahnen kann. Dies gilt namentlich von Operationen, die in Seiten- oder Knieellbogenlage ausgeführt werden (Wendung, Reposition der Nabelschnur, Placentalösung). Es scheint mir von grosser Wichtigkeit zu sein, dass die Hand bei derlei Eingriffen so lange im Uterus bleibe, besonders aber den Halskanal offen erhalte, bis durch geeignete Massregeln vollständige Contraction der Gebärmutter, oder vollständiges Auspressen der etwa aspirirten Luft zu Stande gekommen ist. Demgemäss sollte die Hand niemals in. Seitenlage herausgebracht werden, sondern erst dann, wenn die Kreissende die Rückenlage wieder eingenommen hat; aus denselben Gründen sollte die manuelle Extraction des gewendeten oder ursprünglich mit dem Beckenende vorliegenden Kindes niemals in Seitenlage aus- 
geführt werden. Wenn auch keine anderen Gründe vorlägen, so müsste schon die Gefahr des Lufteindringens das Gebären in Knieellbogenlage oder "in Knieschulterlage verbieten. Aehnliche Bedenken tauchen gegen das Abwarten des Blasensprunges in Seitenlage auf, namentlich in allen Fällen, in denen der vorlicgende Theil den Genitalkanal nicht vollständig und sicher tamponirt, also in erster Linie bei Hydramnios, dann bei Quer- und Beckenendlagen. In vielem gleiche Verhältnisse zur Zeit des Blasensprunges finden sich nicht selten bei engem Becken, ja in gewissem Grade bei den meisten Mehrgebärenden.

Cordwent's Fall illustrirt die Gefahren der Geburt in aufrechter Stellung von einer neuen Seite. Es scheint mir nicht zweifelhaft, dass ähnliche Verhältnisse, eine Art Zurückfederns des Uterus, auch in Rückenlage sich ausbilden können, wenn unter gewaltiger Anstrengung der Bauchpresse präcipitirte Geburt zu. Stande kommt und im Augenblicke des Kindesdurchtrittes der Druck von oben plötzlich sistirt. Ich halte es für praktisch wichtig, auf folgendes Analogon aufmerksam zu machen. Bei forcirter Expression der Nachgeburt (Credé's Methode) geschieht es nicht eben selten, dass nach langem Bemühen endlich der Kuchen plötzlich hervorschiesst, während die Hand dem Uterus'nicht rasch genug folgen kann. Ein ander Mal kommt es vor, das während kräftigen Druckes die ermü,dete Hand vom Gebärmuttergrunde abgleitet, dass demnach der Druck plötzlich sistirt. In beiden Fällen ist ein Zurückschnellen des Uterusgewebes wohl leicht möglich und damit Gelegenheit zur Aspiration gegeben.

Es braucht endlich kaum erwähnt zu werden, dass bei Ansammlung von Fäulnissgasen im Uterus (Physometra) die schleunigste Entleerung des Organes geboten ist. Vielleicht könnte in Fällen, in denen die sofortige Entbindung nicht möglich, das Einführen eines langen, nicht zu weichen oder geradezu harten Rohres (starker englischer oder männlicher Metallkatheter) in das Cavum uteri von Nutzen sein, insofern durch denselben während der Contraction die Gase im Stande wären, nach aussen za entweichen. 